\title{
Control de hongos fitopatógenos asociados a semillas de palto Persea americana Mill. (Lauraceae) In Vitro
}

\author{
Betsabe Leon Ttacca \\ bleonttacca@gmail.com \\ Universidad Nacional de Cañete \\ Lima-Perú \\ Luz Leonor Mattos Calderon \\ leomattos@lamolina.edu.pe \\ Universidad Nacional Agraria La Molina \\ Lima- Perú.
}

\section{RESUMEN}

El trabajo de investigación se realizó en el laboratorio de la Universidad Nacional Agraria La Molina, Perú, como una alternativa al control químico se efectuaron ensayos In vitro para determinar el efecto de los microorganismos Trichoderma harzianum (T), Bacillus subtilis $(B)$ y fertilizante proteinato de cobre $(\mathrm{P})$ para el control de Lasiodioplodia theobromae y Fusarium verticillioides asociados a semillas de palto. Técnicas de cultivos duales, triples y cuádruples se realizaron en medio papa dextrosa agar entre los patógenos y tratamientos ( $\mathrm{T}, \mathrm{B}, \mathrm{P}, \mathrm{T}+\mathrm{B}, \mathrm{T}+\mathrm{P}, \mathrm{B}+\mathrm{P}$ y $\mathrm{T}+\mathrm{B}+\mathrm{P}$ ) bajo un diseño completo al azar con arreglo factorial de 2 (patógenos) x 7 (tratamientos) con cuatro repeticiones. Todos los tratamientos tuvieron efecto en el control de ambos patógenos con valores de inhibición micelial mayor del $60 \%$. La interacción entre T. harzianum + Proteinato de cobre fue el tratamiento con mayor efecto en la inhibición micelial de los patógenos con $91.23 \%$ seguido del fertilizante proteinato de cobre con $87.67 \%$ y $T$. harzianum con $81.84 \%$ de inhibición, en comparación a Bacillus subtilis. que tuvo menor efecto en el control de estos patógenos fungosos.

Palabras clave: Control; Fusarium verticillioides; Lasiodiplodia theobromae; antagonista; aguacate. 


\title{
Control of phytopathogenic fungi associated with avocado seeds Persea americana Mill. (Lauraceae) In Vitro
}

\begin{abstract}
The present study was carried out in the laboratory of the La Molina National Agrarian University, Perú, as an alternative to chemical control through trials of biological control In vitro and greenhouse to determine the effect of the microorganisms Trichoderma harzianum (T), Bacillus subtilis $(B)$ and copper proteinate as fertilizer $(\mathrm{P})$ to control of the patogenic fungi Lasiodioplodia theobromae and Fusarium verticillioides associated with avocado seeds.

Dual, triple and quadruple culture techniques were performed on potato dextrose agar medium between pathogens and treatments $(\mathrm{T}, \mathrm{B}, \mathrm{P}, \mathrm{T}+\mathrm{B}, \mathrm{T}+\mathrm{P}, \mathrm{B}+\mathrm{P}$ and $\mathrm{T}+\mathrm{B}+\mathrm{P})$ under a complete random design with factorial arrangement of 2 (pathogens) $\mathrm{x} 7$ (treatments) with four repetitions. All treatments had effect on the control of both pathogens with more than $60 \%$ mycelial inhibition. The highest values were obtained by the interaction of T. harzianum + copper proteinate with $91.23 \%$ of mycelial inhibition followed by copper proteinate with $87.67 \%$ and $T$. harzianum with $81.84 \%$ in inhibition, compared to Bacillus subtilis, which had the lowest effect on the control of these fungal pathogens.
\end{abstract}

Keywords: Control, Fusarium verticillioides, Lasiodiplodia theobromae, antagonist, avocado.

Artículo recibido: 05 de Abril 2021 Aceptado para publicación: 28 de Mayo 2021 Correspondencia: bleonttacca@gmail.com Conflictos de Interés: Ninguna que declarar 


\section{INTRODUCCIÓN}

La palta o aguacate, Persea americana Mill. (Lauraceae) es una fruta que se encuentra con una tendencia creciente en su producción debido al incremento de la demanda en el mercado mundial, se produce en las regiones tropicales y subtropicales. En el Perú, este cultivo está adaptado a los valles de la Costa, a los valles interandinos de la Sierra y la Selva, sobre todo en la Selva Alta (Mont Koc, 2000).

La producción a nivel mundial muestra un crecimiento de un 5,1\% por año, en el 2017 se ha alcanzado la cifra de producción más elevada con 6,048 mil toneladas. Siendo Perú el tercer país en volumen de producción, con un $8 \%$ de participación, presenta un moderado incremento de su rendimiento de 8,7 toneladas en 2005 pasa a 11,8 toneladas en el 2017. En el 2018 ha logrado colocar al mundo 359 mil toneladas, todo un récord, siendo la Unión Europea el principal mercado de destino, con un 63\% de participación, y los Estados Unidos el segundo en importancia con un 23\% de participación (MINAGRI, 2019)

La principal forma de propagación de este cultivo, es injertando una planta patrón previamente desarrollada a partir de una semilla (Leal-Nares, Gonzales, \& Vidales, 2004). Sin embargo, una serie de enfermedades severas de origen fungoso son diseminadas por las semillas, que en casos extremos provocan la muerte del árbol y, en general, causan una disminución de la producción pudiendo llegar a alcanzar el 14\% y una reducción en la calidad de un $10 \%$, siendo las de mayor importancia económica los causados por hongos fitopatógenos de los géneros Colletotrichum, Dothiorella, Lasiodiplodia, Alternaria, Fusarium, Verticillium, entre otros (Alama, Maldonado, \& Gálvez, 2006; Leal-Nares et al., 2004; León \& Mattos, 2016). Asimismo, las pudriciones de frutos causadas por los hongos fitopatógenos: Lasiodiplodia theobromae, Colletotrichum gloesporioides, Dothiorella sp., Phoma sp., Rhizopus stolonifer, Alternaria sp. y Fusarium sp., son uno de los principales problemas en pre y post-cosecha (Mont Koc, 2000).

El control de las enfermedades producidas por los hongos mencionados está limitado principalmente al uso de fungicidas como oxicloruro de cobre y benomil en precosecha;sin embargo, las aplicaciones con fungicidas tienden a producir residuos visibles sobre la superficie del fruto, los cuales son descartados al momento de la selección. Además, debido al uso prolongado de fungicidas puede conllevar a acumular 
resistencia en los patógenos y al incremento de la concientización poblacional sobre la polución de los pesticidas; por ello, es necesario encontrar nuevas alternativas en las medidas de control, como el control biológico. Considerando que, varias especies de Trichoderma son efectivas en el biocontrol de varios hongos patogénicos de suelo y sólo en algunos casos son recomendados en post-cosecha y manchas foliares; siendo el micoparasitismo, la antibiosis, la competencia por nutrientes y espacio, los mecanismos de acción más importantes de especies de Trichoderma que son utilizados durante el biocontrol (Bailey et al., 2008; Guédez, Cañizález, Castillo, \& Olivar, 2009; Infante, Martínez, González, \& Reyes, 2009). Asimismo, la bacteria Bacillus subtilis produce metabolitos antimicrobiales los cuales inhiben el crecimiento y lisan ciertos hongos filamentosos (Mejía-Bautista et al., 2016; Ortega \& Stalin, 2016). Además, como una alternativa dentro del control químico se tiene la utilización de proteinato de cobre (Promet $\mathrm{Cu}$ ) que induce las defensas naturales de la planta, y en algunos casos afecta directamente el desarrollo del patógeno debido al efecto del cobre (Química Suiza, 2014). Esta situación condujo la realización del trabajo de investigación con el objetivo de evaluar In vitro el efecto del control biológico (B. subtilis y T. harzianum) y químico (proteinato de cobre) de los principales hongos fitopatógenos asociados a semillas de palto usadas para patrón.

\section{ESTRATEGIAS METODOLÓGICAS O MATERIALES Y MÉTODOS}

\section{Ubicación}

El presente trabajo de investigación se realizó en el laboratorio de la especialidad de Fitopatología de la Universidad Nacional Agraria La Molina.

\section{Aislamiento é identificación de hongos fitopatógenos}

Los patógenos fueron aislados de semillas de palto de la raza Mexicana, procedentes de los semilleros de la ciudad de Ayacucho (Luricocha) e identificados por Leon y Mattos (2016). Estos patógenos fueron propagados en medio papa dextrosa agar (PDA) e incubados a $25{ }^{\circ} \mathrm{C}$ por 5 días para realizar los ensayos de control In vitro.

\section{Pruebas de enfrentamientos}

Para esta prueba se realizaron enfrentamientos dobles, triples y cuádruples entre los patógenos y biocontroladores en placas petri $(90$ x 15mm) conteniendo medio PDA según la tabla 1 y figura 01 . Para cada enfrentamiento se obtuvo discos $(0.5 \mathrm{~cm}$ de diámetro $)$ de medio de cultivo con micelio del hongo bicontrolador T. harzianum (Promot) y de los 
hongos fitopatógenos (Lasidioplodia y Fusarium) del margen de cultivos (PDA) de 5 días de edad. Para el caso de B. subtilis se obtuvo un cultivo de 48 horas de edad en medio PDA, donde se adicionó $5 \mathrm{ml}$ de agua destilada estéril, y con la ayuda de un ansa de kolle embebida en la suspensión, se realizó una raya de 30mm en el extremo de la placa sobre el medio PDA. Para el proteinato de cobre (Promet $\mathrm{Cu}$ ) se preparó una mezcla del producto en $100 \mathrm{ml}$ de agua (2,5 ml/litro); asimismo, se preparó discos de cartulina estériles (1 $\mathrm{cm}$ de diámetro) que fueron sumergidos en la suspensión de proteinato por 1 minuto, los discos fueron colocador en el extremo de la placa sobre el medio PDA. Para determinar el porcentaje de inhibición del crecimiento micelial de los patógenos. Se registró medidas del crecimiento radial de la colonia $(\mathrm{cm})$ a los 7 y 10 días para los tratamientos con enfrentamiento triples y cuádruples, y doble respectivamente (Sivakumar et al., 2000).

Tabla 1. Tratamientos para el control de patógenos de semillas de palto.

\begin{tabular}{ccl} 
Tratamientos & Código & \multicolumn{1}{c}{ Ingrediente activo } \\
& & \\
\hline 1 & $\mathrm{~T}$ & T. harzianum \\
2 & $\mathrm{~B}$ & Bacillus subtilis \\
3 & $\mathrm{P}$ & Proteinato de cobre \\
4 & $\mathrm{~T}+\mathrm{B}$ & T. harzianum + Bacillus subtilis \\
5 & $\mathrm{~T}+\mathrm{P}$ & T. harzianum + Proteinato de cobre \\
6 & $\mathrm{~B}+\mathrm{P}$ & Bacillus subtilis + Proteinato de cobre \\
7 & $\mathrm{~T}+\mathrm{B}+\mathrm{P}$ & T. harzianum + Bacillus subtilis + \\
& & Proteinato de cobre \\
\hline
\end{tabular}

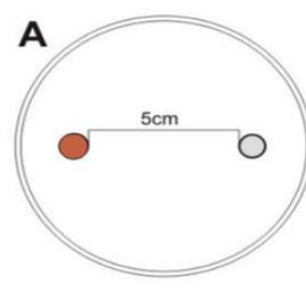

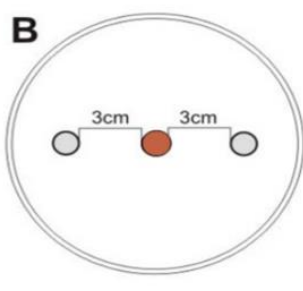

Patógeno
C

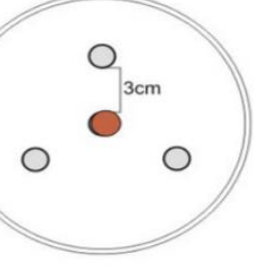

D

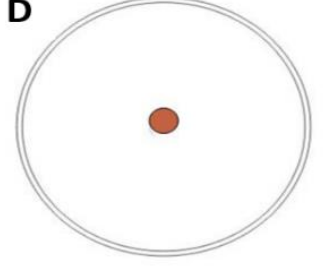

Tratamiento

Figura 1. Croquis de siembra para la prueba de control biológico in vitro: A. cultivo dual; B. cultivo triple; C. cultivo cuadruple D. Control, para las pruebas. 


\section{Análisis estadístico}

El diseño estadístico que se empleó fue el diseño completamente al azar (DCA) con arreglo factorial de 2 × 7; siendo, los factores patógenos y tratamientos biológicos y químicos. Se trabajó con un total de 14 tratamientos y 4 repeticiones, la variable de respuesta que se evaluó fue el porcentaje de inhibición del crecimiento micelial de los patógenos. Con los datos se realizó el análisis de variancia (ANOVA) y prueba de contraste de Duncan $(\mathrm{P}=0,05)$.

\section{RESULTADOS Y DISCUSIÓN}

El análisis de varianza (tabla 2) indica que no hubo diferencias significativas para los patógenos $L$. theobromae y $F$. verticillioides; no obstante, existe significancia en los tratamientos empleados para el control de los patógenos e interacción de patógenos y tratamientos $((\mathrm{p}<=0.05)$. En la Tabla 3 y figura2, se muestra el efecto de los diferentes tratamientos en la inhibición del crecimiento micelial de los fitopatógenos. Todos los controladores tuvieron valores de inhibición micelial mayor del 60\%. Las interacciones de T. harzianum + Proteinato de cobre presentaron los mayores valores de porcentaje de inhibición promedio para ambos patógenos con $91.23 \%$, seguido de Proteinato de cobre con 87, $67 \%$; sin embargo, en los tratamientos con T. harzianum (con 81,84\%) e interacciones de T. harzianum + B. subtilis + Proteinato de cobre (con 80,56\%) no existen diferencias significativas. Mientras que, los valores más bajos lo obtuvieron los tratamientos B. subtilis con $62.88 \%$, e interacciones de T. harzianum + B. subtilis con $68,2 \%$.

\section{Tabla 2. Análisis de varianza del efecto de tratamientos biológicos y químicos en el} control de L. theobromae y F. verticillioides

\begin{tabular}{llllll}
\hline \multicolumn{1}{c}{ F.V. } & SC & Gl & CM & F & p-valor \\
\hline Modelo & 5328.96 & 13 & 409.92 & 91.53 & $<0.0001$ \\
Patógenos & 13.95 & 1 & 13.95 & 3.12 & 0.0848 \\
Tratamientos & 5058.2 & 6 & 843.03 & 188.25 & $<0.0001$ \\
Patógenos* & & & & & \\
Tratamiento & 256.81 & 6 & 42.8 & 9.56 & $<0.0001$ \\
Error & 188.09 & 42 & 4.48 & & \\
Total & 5517.05 & 55 & & & \\
\hline
\end{tabular}

$\mathrm{R}^{2}$ : 0.97; CV: 2.71 
Tabla 3. Porcentaje de inhibición micelial de Lasiodiplodia theobromae y Fusarium verticillioides

\begin{tabular}{cc}
\hline Tratamientos & $\begin{array}{c}\text { Inhibición micelial } \\
(\%)\end{array}$ \\
\hline $\mathrm{T}+\mathrm{P}$ & $91.23 \mathrm{a}$ \\
$\mathrm{P}$ & $87.67 \mathrm{~b}$ \\
$\mathrm{~T}$ & $81.84 \mathrm{c}$ \\
$\mathrm{T}+\mathrm{B}+\mathrm{P}$ & $80.56 \mathrm{c}$ \\
$\mathrm{B}+\mathrm{P}$ & $73.78 \mathrm{~d}$ \\
$\mathrm{~T}+\mathrm{B}$ & $68.2 \mathrm{e}$ \\
$\mathrm{B}$ & $62.88 \mathrm{f}$ \\
\hline
\end{tabular}

Datos promedio de inhibición micelial de los patogógenos Lasiodiplodia theobromae y Fusarium verticillioides. $\mathrm{T}=$ Trichoderma harzianum; $\mathrm{B}=$ Bacillus subtilis; $\mathrm{P}=$ Proteinato de cobre. $\mathrm{T}+\mathrm{B}$ y $\mathrm{B}+\mathrm{P}$ : Enfrentamiento dual. $\mathrm{T}+\mathrm{B}+\mathrm{P}$ : Enfrentamiento triple. Letras diferentes indican diferencias significativas $(p<=0.05)$. Fuente: Elaboración propia

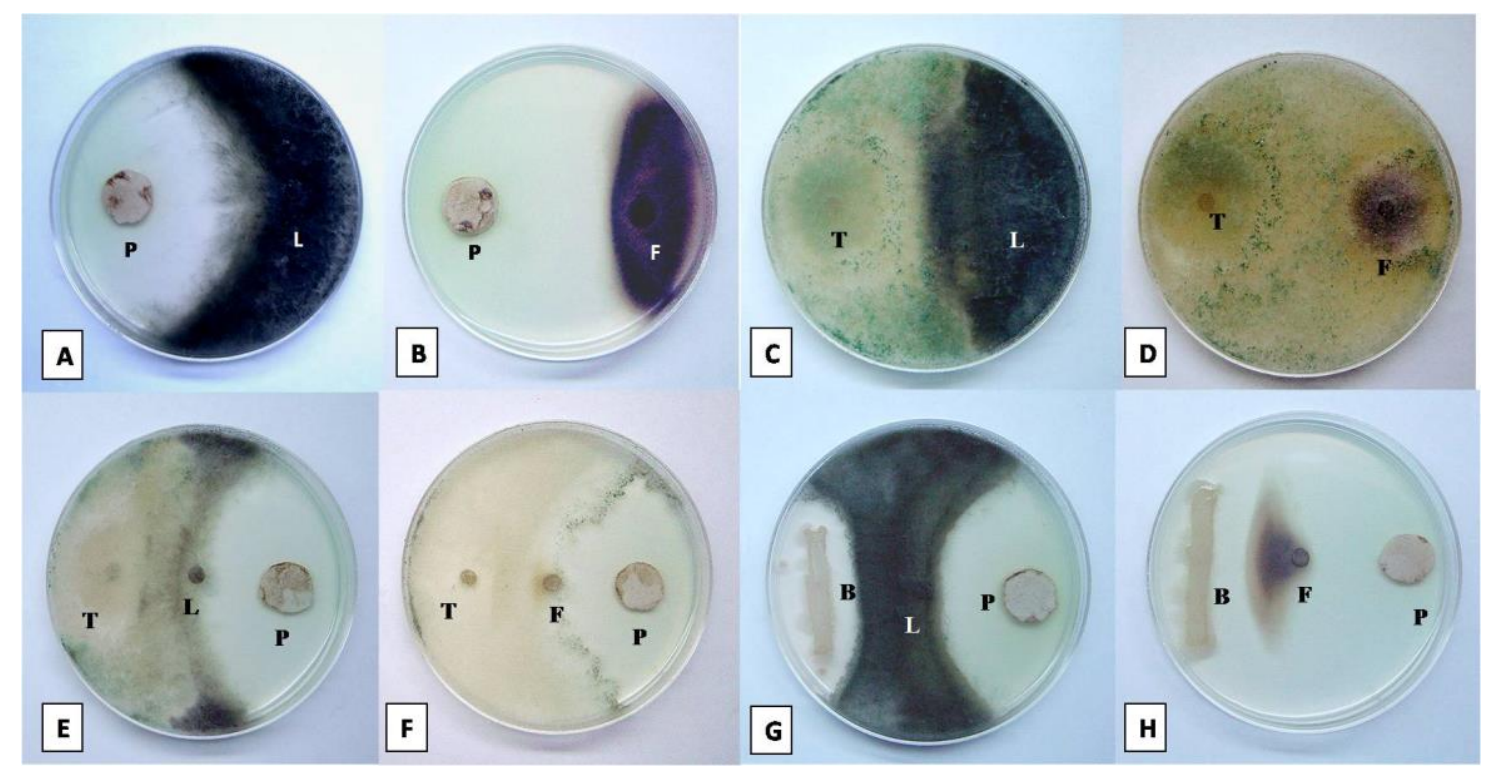

Figura 2. Efecto de los controladores en el crecimiento micelial de Lasiodiplodia theobromae (L) y Fusarium verticillioides (F). A y B. Enfrentamiento dual entre Proteinato de cobre y patógenos. C y D. Enfrentamiento dual entre Trichoderma harzianum y patógenos. E y F. Enfrentamientos triples entre Trichoderma harzianum, Proteinato de cobre y patógenos. G y H. Enfrentamientos triples entre Bacillus subtilis, Proteinato de cobre y patógenos.

\section{Fuente: Elaboración propia}


Ademas, en la interacción patógenos y controladores, la mayor inhibición micelial se dio en el patógeno Fusarium verticillioides con el enfrentamiento triple entre $T$. harzianum y proteinato de cobre ( $95.91 \%$ ), seguido del enfrentamiento dual con proteinato de cobre (87.84\%) ; así mismo, para el patógeno L. theobromae la mayor inhibición micelial se presentó con los mismos tratamientos con 86.55 y $87.5 \%$ respectivamente. No obstante, los valores más bajos lo obtuvieron los tratamientos con B. subtilis con $60.14 \%$ y 65.63 $\%$ de inhibición micelial de Fusarium verticillioides y Lasiodiplodia theobromae respectivamente, e interacciones de $T$. harzianum + B. subtilis con 67.73 y $68.67 \%$ respectivamente (Figura 3 ).

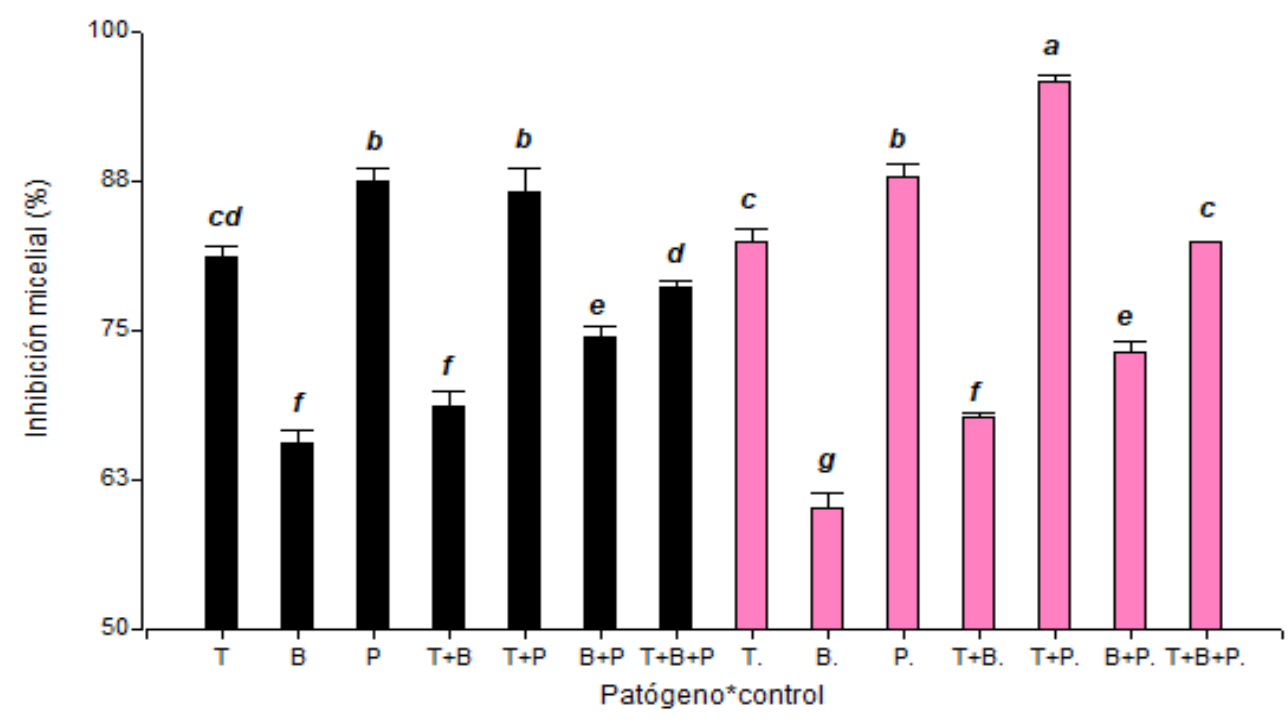

Figura 3. Efecto de los controladores en el crecimiento micelial de Lasiodiplodia theobromae (barras de color negro) y Fusarium verticillioides (barras de color rosado). $\mathrm{T}=$ Trichoderma harzianum $; \mathrm{B}=$ Bacillus subtilis $; \mathrm{P}=$ Proteinato de cobre. $\mathrm{T}+\mathrm{B}$ y B+P: Enfrentamiento dual. T+B+P: Enfrentamiento triple.

Letras diferentes indican diferencias significativas $(\mathrm{p}<=0.05)$.

\section{Fuente: Elaboración propia}

En los resultados obtenidos en la prueba de biocontrol in vitro, se observó que todos los tratamientos tuvieron efecto en la inhibición micelial de los patógenos $F$. verticillioides y L. theobromae. Siendo los tratamientos con enfrentamientos triples de los patógenos contra T. harzianum y proteinato de cobre (Promet $\mathrm{Cu}$ ) con mayor inhibición micelial, seguido del enfrentamiento dual con Proteinato de cobre. Esto probablemente ocurre porque el proteinato actúa directamente sobre el desarrollo de los patógenos por contener 
cobre en su composición (Química Suiza, 2005). Es así que enmiendas de fertilizantes y pesticidas conteniendo $\mathrm{Cu}$ pueden proveer control efectivo de algunas enfermedades estimulando mecanismos de defensa de la planta contra la infección, la producción de componentes antimicrobiales y la resistencia de enfermedad (Lawrence, Wade, \& Don, 2007). Ademas, el proteinato de cobre induce mayor formación de fenolasas de las que normalmente se produce dentro de la planta (Azcon-Bieto, 2000; Química Suiza, 2005), se sabe que las polifenoloxidasas catalizan las reacciones de oxidación de los fenoles de plantas en las paredes celulares, comprometido en la biosíntesis de la lignina y la formacion de melanina (Lawrence et al., 2007). Mientras que, Trichoderma spp. produce y secreta metabolitos antifúngicos, además de enzimas líticas tales como: Quitinasas y $\beta$ -1,3 glucanasas (G. E. Harman, 2006; Mukherjee et al., 2012), a su vez promueven el crecimiento y rendimiento del cultivo, mediante el aumento de la absorción de nutrientes o por la producción de factores de crecimiento como el ácido indolacetico (G. Harman, 2011).

Además, T. harzianum antagoniza a los fitopatógenos por competencia, parasitismo, antibiosis ó por una combinación sinérgica de estos modos de acción (G. E. Harman, 2006; Infante et al., 2009; Mukherjee et al., 2012). En cuanto a las interacciones de los biocontroladores con el proteinato de cobre, se puede decir que muestran un efecto sinérgico, por lo que se podría recomendar aplicaciones de las interacciones de $T$. harzianum + proteinato de cobre en campo. Sin embargo, el cobre tiene un mayor efecto negativo sobre el crecimiento de las bacterias que de los hongos (Huysman, Verstraete, \& Brookes, 1994), como B. subtilis es una bacteria probablemente ha sido afectada por el elemento químico mencionado; por otro lado, en algunos trabajos de investigacion indican que la combinación directa de formulaciones de cobre y B. subtilis en un mismo tanque puede tener efecto inhibitorio sobre este último (Demoz \& Korsten, 2006; VanZwieten, Merrington, \& Van-Zwieten, 2004) También puede deberse a que el control biológico es más influenciado por los factores ambientes que el control químico (Hall \& Menn, 1999). Por consiguiente, la eficacia y consistencia del control biológico se mejora con asociaciones microbiales, con modos de acción complementarios e incluso sinérgicos (Hall \& Menn, 1999; Stewart, Brownbridge, Hill, \& Jackson, 2010).

Por lo general, B. subtilis produce metabolitos los cuales inhiben el crecimiento y causan lisis celular en ciertos hongos filamentosos (Ortega \& Stalin, 2016; Ruiz-Sánchez, Mejía- 
Bautista, Cristóbal-Alejo, Valencia-Botín, \& Reyes-Ramírez, 2014), además dicha bacteria a sido estudiado por su capacidad de proteger a las plantas de sus patógenos y estimular el crecimiento y mejorar el rendimiento del cultivo (Gardener, 2010). Asu vez, B. subtilis se encuentra reportada como agente biocontrolador de enfermedades postcosecha en palto.

\section{CONCLUSIÓN O CONSIDERACIONES FINALES}

En el control In vitro, la mayor inhibición micelial de los patógenos $F$. verticillioides y $L$. theobromae se dio en las interacciones entre Proteinato de cobre $+T$. harzianum, seguido del enfrentamiento dual con proteinato de cobre y $T$. harzianum; mientras que, los tratamientos con menor efecto fueron B. subtilis + proteinato de cobre y B. subtilis.

\section{LISTA DE REFERENCIAS}

Alama, I., Maldonado, E., \& Gálvez, E. R. (2006). Lasiodiplodia theobromae afectando el cultivo de palto (Persea americana) en las condiciones de Piura-Perú. Universalia, 11(2), 4-13.

Azcon-Bieto, J. (2000). Fundamentos de Fisiología Vegetal. Barcelona, ES.: Universidad de Barcelona. .

Bailey, B. A., Bae, H., Strem, M. D., Crozier, J., Thomas, S. E., Samuels, G. J., . . . Holmes, K. A. (2008). Antibiosis, mycoparasitism, and colonization success for endophytic Trichoderma isolates with biological control potential in Theobroma cacao. Biological Control, 46(1), 24-35. doi: http://dx.doi.org/10.1016/j.biocontrol.2008.01.003

Demoz, B. T., \& Korsten, L. (2006). Bacillus subtilis attachment, colonization, and survival on avocado flowers and its mode of action on stem-end rot pathogens. Biological Control, 37(1), 68-74.

Gardener, B. B. M. (2010). Biocontrol of plant pathogens and plant growth promotion by Bacillus Recent developments in management of plant diseases (pp. 71-79): Springer.

Guédez, C., Cañizález, L., Castillo, C., \& Olivar, R. (2009). Efecto antagónico de Trichoderma harzianum sobre algunos hongos patógenos postcosecha de la fresa (Fragaria spp). Revista de la Sociedad Venezolana de Microbiología, 29, 34-38.

Hall, F. R., \& Menn, J. J. (1999). Biopesticides: use and delivery (Vol. 5): Humana Press $\wedge$ eNueva Jersey Nueva Jersey. 
Harman, G. (2011). Trichoderma—not just for biocontrol anymore. Phytoparasitica, 39(2), 103-108.

Harman, G. E. (2006). Overview of Mechanisms and Uses of Trichoderma spp. Phytopathology, 96(2), 190-194. doi: 10.1094/PHYTO-96-0190

Huysman, F., Verstraete, W., \& Brookes, P. (1994). Effect of manuring practices and increased copper concentrations on soil microbial populations. Soil Biology and Biochemistry, 26(1), 103-110.

Infante, D., Martínez, B., González, N., \& Reyes, Y. (2009). Mecanismos de accion de Trichoderma frente a hongos fitopatogenos. Revista de Protección Vegetal, 24, $14-21$.

Lawrence, E., Wade, H., \& Don, M. ( 2007). Mineral and Plant Disease. St.Paul, Minesota, USA.: The American Phytophatological Society.

Leal-Nares, O., Gonzales, E., \& Vidales, I. (2004). Embriogenesis somática por el cultivo in Vitro de tejido nucelar de frutos inmaduros de aguacate (Persea americana Mill.) cv. Hass. Morelia, MX. Instituto de investigaciones Químico-Biológicas, UMSNH.

León, B., \& Mattos, L. (2016). Hongos Fitopatógenos asociados a semillas de palto (Persea Americana mill.). Revista Investigaciones Altoandinas, 18(4), 423-430.

Mejía-Bautista, M. Á., Reyes-Ramírez, A., Cristóbal-Alejo, J., Tun-Suárez, J. M., del Carmen Borges-Gómez, L., \& Pacheco-Aguilar, J. R. (2016). Bacillus spp. en el Control de la Marchitez Causada por Fusarium spp. en Capsicum chinense. Revista Mexicana de Fitopatología, Mexican Journal of Phytopathology, 34(3).

Mont Koc, R. (2000). El palto y sus enfermedades. Lima, PE.: SENASA.

Mukherjee, M., Mukherjee, P., Horwitz, B., Zachow, C., Berg, G., \& Zeilinger, S. (2012). Trichoderma-Plant-Pathogen Interactions: Advances in Genetics of Biological Control. Indian Journal of Microbiology, 52(4), 522-529. doi: 10.1007/s12088012-0308-5

Ortega, N., \& Stalin, E. (2016). Uso de la bacteria bacillus subtilis como agente de control biológico de hongos fitopatógenos en cultivos tropicales. Machala: Universidad Técnica de Machala.

Química Suiza. (2005). Promet Cu: Inductor de fenolasas. In Q. Suiza (Ed.), (pp. 5). Lima. Perú. 
Ruiz-Sánchez, E., Mejía-Bautista, M. Á., Cristóbal-Alejo, J., Valencia-Botín, A., \& Reyes-Ramírez, A. (2014). Actividad antagónica de filtrados de Bacillus subtilis contra Colletotrichum gloeosporioides (Penz.). Revista mexicana de ciencias agrícolas, 5(7), 1325-1332.

Stewart, A., Brownbridge, M., Hill, R. A., \& Jackson, T. A. (2010). Utilizing soil microbes for biocontrol Soil Microbiology and Sustainable Crop Production (pp. 315-371): Springer.

Van-Zwieten, L., Merrington, G., \& Van-Zwieten, M. (2004). Review of impacts on soil biota caused by copper residues from fungicide application. SuperSoil, 2004, 3rd. 